Rasol, K.; Domingo, J. Multi-level hierarchical controller placement in software defined networking. A: International Networking Conference. "Selected Papers from the 12th International Networking Conference. INC 2020". Berlín: Springer, 2020, p. 131-145. ISBN 978-3-030-64757-5.

The final authenticated version is available online at https://doi.org/10.1007/978-3-030-64758-2_10

\title{
Multi-level Hierarchical Controller Placement in Software Defined Networking
}

\author{
Kurdman Abdulrahman Rasol ${ }^{1}$ and Prof. Jordi Domingo-Pascual ${ }^{2}$ \\ ${ }^{1}$ Department of Computer Architecture, Universitat Politècnica de Catalunya \\ (UPC), Barcelona, Catalonia, Spain, kurdman.rasol@upc.edu \\ 2 Department of Computer Architecture, Universitat Politècnica de Catalunya \\ (UPC), Barcelona, Catalonia, Spain, jordi.domingo@ac.upc.edu
}

\begin{abstract}
Software Defined Networking (SDN) is a widely used network architecture. It separates the controller logic (or control plane) from forwarding plane (or data plane) to manage the whole network and it enables the network scalability and programmability. One of the most significant challenges in Software Defined Networking (SDN) is the Controller Placement Problem (CPP), which tries to specify the minimum number of controllers and their optimal location.

In our study, we extend the methodology based on K-means and Kcenter algorithms to solve the Controller Placement problem (CPP) into a Multi-level Hierarchical Controller Placement Problem (HCPP), where the Super Controller (SC) is in the top-level, some Master Controllers (MCs) are in the intermediate level and the Domain Controllers (DCs) are at the bottom level. The optimization metric is the latency between controller and switches assigned to it and the latency for controller to controller communication.

The proposed architecture and methodology is applied on Western European NRENs topology taken from Internet Topology Zoo. The entire network topology is divided into small scale networks (Clusters) and for each cluster, the optimal number of controllers (Domain Controllers) and their placement is found. A second optimization identifies the optimal number of Master Controllers and their optimal placement.

The results validate the methodology and show its feasibility on large networks and different domains. A useful use case may be the deployment of hierarchical levels of controllers for the enforcement of very precise routing policies through different domains.
\end{abstract}

Keywords: Software Defined Network · Controllers Placementd · Latency Optimization · Hierarchical Control Plane · Multistage Optimization

\section{INTRODUCTION}

The key idea of Software Defined Networking (SDN) is separation the forwarding plane (or data plane) and the control plane to manage network performance. SDN feasibility is supported by a logically centralized control plane [1] [2]. 
The main focus of this paper is to minimize the control plane latency. Control latency comprises transmission delay, propagation delay, switch queuing latency and controller processing latency [3] [4]. The transmission delay at the switch and controller refers to the ratio of packet size and the data rate of a link. The propagation delay depends on the distance between switches and controllers. The queuing latency is mainly determined by congestion in the path between source and destination. The controller processing latency is principally influenced by the load of the controller. In our current research, the metric of the optimization study is based on propagation delay and referred as latency.

The placement of the controller is a key design choice of the SDN control plane. The controller placement problem (CPP) is a non-deterministic polynomial NP-hard problem and is similar to the facility location problem [5]. Heller et al [6] had proposed the principal objective to solve the Controller Placement Problem (CPP) by minimizing the propagation delay (latency) to compute the optimum location of the controllers deployed and the minimum number of controllers to be placed. The authors conducted experiments on the Internet2 OS3E network topology [7]. K-means or K-median is defined for minimizing average propagation delay [8], while K-center is for minimizing the largest distance latency between controllers and their associated switches [9]. For finding the optimum controller positions of the networks, the following questions must be solved:

1. What should be the minimum number of the controller to be placed in a network?

2. Where to place the controllers?

In this paper, we consider the scenario of Uncapacitated Controller Placement Problem (UCPP) [10]. This means that the capacity is not considered as a constraint and the controller is located with a switch [11].

Deploying a single controller is inefficient to manage a large network. Placing multiple controllers is still an outstanding challenge. For large size networks, multiple controllers are most efficient to handle the control plane traffic in order to improve the scalability and latency of the network [12]. Kuang et al.[13] proposed an algorithm based on a hierarchical K-means algorithm to solve a controller placement problem. The objective of the study is to minimize the maximum latency between the controller and its associated switches and to balance the load of each controller. The main contribution of the work is: a) the large network is divided into several single controller domains. b) In the process of network partitioning, not only latency between the switches and controller is taking into account but also the load balancing. The experimental results illustrate the partitioning used $\mathrm{K} *$-mean algorithm it is proven to be more balanced than the optimized K-means algorithm but the propagation latency is larger than the optimized K-means algorithm by Wang et al [8].

For very large networks composed of several Autonomous Systems (AS), we explore the feasibility of a Multi-level Hierarchical Control Plane. The multi-level hierarchical controller plane approach is efficient because the controller-controller latencies must be considered along with the controller-switch latencies when evaluating the control plane reactivity perceived by the switches. The traffic on the 


\begin{tabular}{||cc||}
\hline Symbol & Description \\
\hline \hline$G(V, E, L)$ & Physical Network \\
$V$ & Set of switches in the network (nodes) \\
$E$ & Set of physical links between switches (edges) \\
$L$ & Set of switch locations \\
$C$ & Set of Controllers, where $(c \subset V)$ \\
$(i, j)$ & The link between node $i$ and node $j$ \\
$d(v, c)$ & The distance between switch $v \in V$ and controller $c \in C$. \\
$n$ & Total number of switches or nodes in the network, where $(n=|V|)$ \\
$k$ & Total number of controllers to be installed in the network, where $(k \leq n)$ \\
$P$ & Set of all possible placement for $k$ controllers \\
$w_{k}$ & Indicates whether a controller is deployed at location $k(=1)$ or not $(=0)$ \\
$x_{i, k}$ & Indicates whether switch $i$ is connected to controller $k(=1)$ or not $(=0)$ \\
\hline
\end{tabular}

controller-controller plane is crucial to achieving a consistent shared view of the network state that is the required condition to run network applications and the network state is stored in shared data structures. The response time of packets transmitted from the controller to each associated switches or from the switch to the controller is usually shorter than in the controller-controller traffic communication. In general, the controller-controller traffic is very complex because distributed controllers adopt coordination protocols and algorithms to synchronize their shared data structures to guarantee a consistent global network view and to enable a centralized view of the network state for the applications. The controller-switch traffic in control plane fundamentally depends on the network application running on the controller. As a result, the optimal controller placement in a given network should consider both kinds of latencies. On the other hand, our work concentrates on the controller placement problem by studying the impact of both latencies.

\section{General Formulation of the Controller Placement Problem}

The network is modelled as a graph $G=(V, E, L)$, where $V$ represents the set of switches, $E$ represents the set of links between switches and $L$ represents the set of switch locations (longitude and latitude). To formulate the problem mathematically, Lavg denotes the average latency. Let $d(v, c)$ be the distance (or edge weights) between switches $v \in V$ and the controllers $c \in C$. The model optimizes the latency between switches and controllers. It is also important to note that the number of controllers must be less than or equal to the number of switches $n=|V|$. The controller is deployed in the location of a switch. The number of controllers is fixed to $k$ where $k \leq n$. The set of controllers to be installed is represented as $C=\left\{c_{1}, c_{2}, c_{3}, \ldots, c_{k}\right\}$. Therefore, the set of all possible placements $(\mathrm{P})$ can be represented as $P=\left\{P_{1}, P_{2}, P_{3}, \ldots, P_{m}\right\}$, where $m$ denotes the variations of $n$ elements taken in groups of $k$ without repetitions. 
The number of all possible placements $(m)$ can be calculated as:

$$
m=\frac{n !}{k !(n-k) !}
$$

A reference study for the Controller Placement Problem in Software Defined Networking was done by Heller [6]. In the CPP, one of the most frequently utilized performance metrics is latency (or delay). Generally, latency describes the total time taken by a packet from the source node to the destination node. In this work, we take the latency as the distance between two nodes. In this paper, we study two types of optimization metrics which are average-case latency and worst-case latency [6].

\subsection{Average-Case Latency:}

The average latency for the placement of the controller minimizes the average distances between the controller and switches assigned to the controller. This optimization problem is known as the minimum k-median problem or k-means problem [8]. The objective function can be defined as the follows:

$$
L_{c s-a v g}\left(P^{\prime}\right)=\frac{1}{n} \sum_{v \in V} \min _{\left(c \in P^{\prime}\right)} d(v, c)
$$

Where $d(v, c)$ is the distance between switch $v \in V$ associated with controller $c$. The objective is to find the optimal placement of controller $P^{\prime}$ from the set of all possible controller placements where $\left|P^{\prime}\right|=k$ and the $L_{c s-a v g}\left(P^{\prime}\right)$ is the minimum.

\subsection{Worst-Case Latency:}

The worst-case latency minimizes the worst (or maximum latency) between a switch $v \in V$ and its controller $c \in C$. This optimization problem is known as minimum k-center problem [9]. The k-center problem is a location analysis problem related to the optimization problem in the area of operation research. The $\mathrm{k}$-center scheme provides an optimal solution minimizing the longest distance among nodes. The objective function is defined as follows:

$$
L_{c s-w o r s t}\left(P^{\prime}\right)=\max _{v \in V} \min _{c \in P^{\prime}} d(v, c)
$$

The optimization result comes from minimizing the previous optimization function. The goal of the optimization is to find the optimal minimum-latency placements $P^{\prime}$ from the set of all possible placements $P$. 


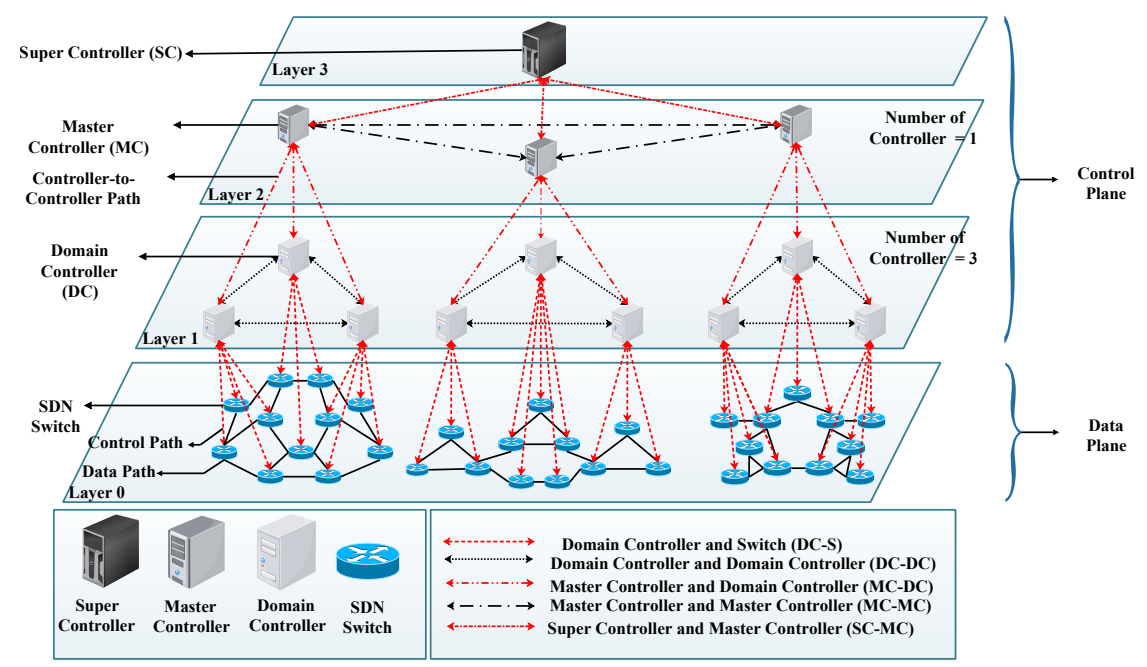

Fig. 1. Multi-level Hierarchical Control Plane Architecture

\subsection{Formulation Constraints:}

The mathematical constraints are computed as follows:

$$
\begin{array}{r}
\forall i \in V: \sum_{k \in P} x_{i, k}=1 \\
\sum_{k \in P} w_{k}=k \\
\forall i \in V, \forall k \in P: x_{i, k} \leq w_{k} \\
\forall k \in P: w_{k} \in\{0,1\} \\
\forall i \in V, \forall k \in P: x_{i, k} \in\{0,1\}
\end{array}
$$

Equation (4) ensures that each switch is associated with exactly one controller. Equation (5) restricts that the number of controller deployed is exactly to $k$. (6) signifies that switch $i$ is located to controller $k$ if controller $k$ deployed onto switch $i$. Constraints (7) and (8) specify that the variables $x_{i, k}$ and $w_{k}$ are binary (equal to 0 or 1 ).

\section{Proposed Architecture and Methodology}

The methodology proposed is named Multi-level Hierarchical Controller Placement Problem (HCPP). In the hierarchical architecture, the controller at the higher-level manages the controllers at the lower-level. There are at least two levels of controllers and it splits the control plane into multiple levels. The advantage of the multi-level architecture improves the scalability and the efficiency 
of the network [14]. Ideally, a hierarchical approach may support any number of levels but for each scenario, the optimal number should be found. Fig.1 illustrates the Multi-level Hierarchical Control Plane Architecture with three controller layers, where the Super Controller (SC) is in the top-level, some Master Controllers (MC) are in the intermediate level and the Domain Controllers (DC) at the bottom level.

There are five different types of communications: Domain Controller and Switch (DC-S), Domain Controller and Domain Controller (DC-DC), Master Controller and Domain Controller (MC-DC), Master Controller and Master Controller (MC-MC) and Super Controller and Master Controller (SC-MC). This architecture requires three controller layers and thus the optimization of three-phases:

1. First Optimization: At the first stage of optimization, the latency between switches and Domain Controllers (DCs) is minimized. All Domain Controllers (DCs) are at the one level and responsible to manage switches in the domain.

2. Second Optimization: The goal of the second optimization is to identify the number and placement of Master Controllers (MCs) where the Domain Controllers (DCs) act as switches (controlled devices). The topology for connecting Domain Controllers (DCs) is built as a virtual network (VN) based on the physical underlying topology and computing the shortest path between pairs of Domain Controllers (DCs).

Dijkstra Algorithm is implemented on the top physical underlying topology to determine the shortest path for all pairs of Domain Controllers (DCs).

3. Third Optimization: In the upper-level, a super controller (SC) acts as a global controller which is logically centralized control plane. Again, a virtual topology connecting all Master Controllers (MCs) is built based on the physical underlying topology. Then, using Dijkstra Algorithm to compute the shortest path between all pairs of Master Controllers (MCs) to find the placement of the Super Controller (SC).

\section{Evaluation and Results}

\subsection{Western European NRENs Topology}

The proposed method to solve the Hierarchical Controller Placement Problem (HCPP) is applied on a set of networks of Western European National Research and Education Network NRENs (278 routers) shown in Fig.2(left). The data about Western European NREN is obtained from Internet Topology Zoo [15].

We define each country as a cluster except the countries with less than six nodes, which are integrated with the nearest country. This foot tries to consider the real constraints derived from each network having its own management agency.

The data from Internet Topology Zoo presents seven nodes which are identified only by the name of the city or a country. We completed the database with 

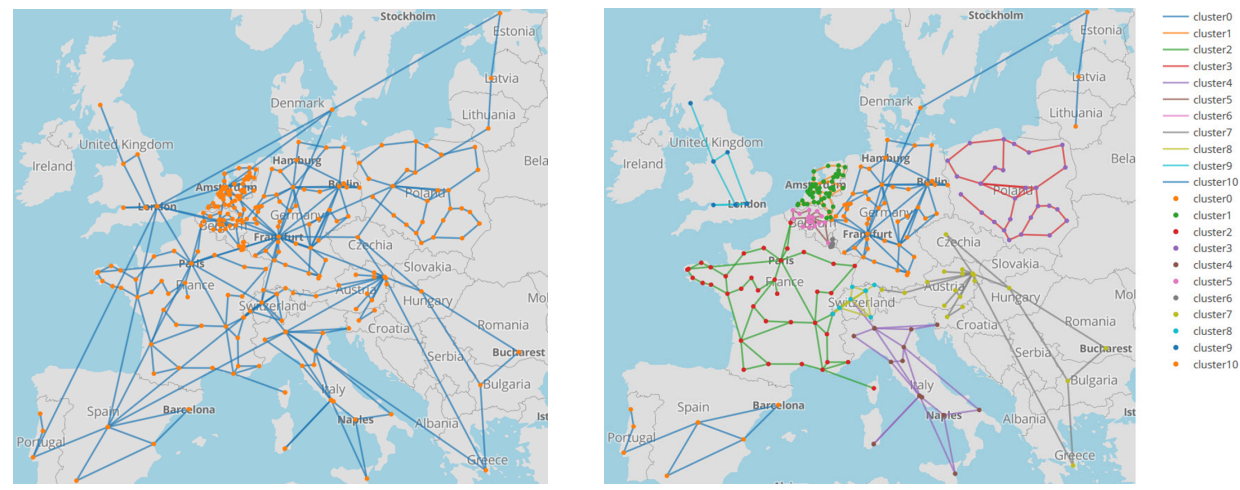

Fig. 2. The entire Western European NRENs Network Topology (left) and Western European NRENs Network Topology with Clusters (right)

Table 1. Cluster List

\begin{tabular}{|c|c|c|c|}
\hline Country & Cluster & Nodes & Network \\
\hline Germany & 0 & 52 & DFN \\
\hline Denmark & 0 & 1 & GEANT \\
\hline Estonia & 0 & 1 & GEANT \\
\hline Latvia & 0 & 1 & GEANT \\
\hline Lithuania & 0 & 1 & GEANT \\
\hline Netherlands & 1 & 51 & SURFnet \\
\hline France & 2 & 39 & RENATER \\
\hline Poland & 3 & 28 & PSNC \\
\hline Italy & 4 & 20 & GARR \\
\hline Belgium & 5 & 19 & BELnet \\
\hline Luxembourg & 6 & 15 & RESTENA \\
\hline Austria & 7 & 13 & ACOnet \\
\hline Slovenia & 7 & 2 & ARNES \\
\hline Czech Republic & 7 & 1 & GEANT \\
\hline Hungary & 7 & 1 & GEANT \\
\hline Bulgaria & 7 & 1 & GEANT \\
\hline Romania & 7 & 1 & GEANT \\
\hline Greece & 7 & 1 & GEANT \\
\hline Switzerland & 8 & 12 & SWITCH \\
\hline United Kingdom & 9 & 9 & JANET \\
\hline Spain & 10 & 6 & RedIris \\
\hline Portugal & 10 & 3 & FCCN \\
\hline Total & 11 Cluster & 278 Nodes & \\
\hline
\end{tabular}

corresponding coordinates of the city or the capital city of the country. In order to get the coordinates of the city, we checked with Google Maps and obtained its longitude and latitude.

Furthermore, we found out that some of the nodes are exactly in the same geographical location. The optimization results may select either of these locations.

The Western European network is divided into eleven clusters by using a country-based approach (see Fig.2(right). The reason for the partitioned network is to supervise the management of the large network. The clusters are listed on Table1. 
The linearization approach is implemented to transform the problem into mixed-integer programming (MIP) are solved optimally by using the well-know optimizer tool IBM ILOG CPLEX Optimization Studio V12.8.0 [16]. CPLEX is used to provide all optimal solutions and the model is programmed in Python.

The computing time varies from tenths of seconds to a few tens of seconds. The optimization running time to minimize the worst-case latency takes more time than the average-case latency but this is not relevant as it is an offline optimization.

Table 2. OPTIMAL DOMAIN CONTROLLER LOCATION FOR AVERAGE-CASE LATENCY AND WORST-CASE LATENCY

\begin{tabular}{|c|c|c|c|}
\hline \multirow{4}{*}{$\begin{array}{c}\text { Clusters } \\
\text { Co }\end{array}$} & \multicolumn{3}{|c|}{ AVERAGE- CASE LATENCY } \\
\hline & $K=1$ & $K=2$ & $\quad \mathbf{K}=\mathbf{3}$ \\
\hline & Frankfurt & Frankfurt, Potsdam & Frankfurt, Potsdam, Riga \\
\hline & $0.0975 \mathrm{~ms}$ & $0.0736 \mathrm{~ms}$ & $0.0563 \mathrm{~ms}$ \\
\hline C1 & $\begin{array}{l}\text { Utrecht } \\
0.0280 \mathrm{~ms}\end{array}$ & $\begin{array}{c}\text { Hoogeveen, Utrecht } \\
0.0211 \mathrm{~ms}\end{array}$ & Hoogeveen, Utrecht, Breda \\
\hline C2 & Paris & Nantes, Lyon & Paris, Nantes, Lyon \\
\hline & $0.1245 \mathrm{~ms}$ & $0.0900 \mathrm{~ms}$ & $0.0653 \mathrm{~ms}$ \\
\hline C3 & $\begin{array}{c}\text { Lodz } \\
0.0873 \mathrm{~ms}\end{array}$ & $\begin{array}{c}\text { Gdansk, Krakow } \\
0.0650 \mathrm{~ms}\end{array}$ & $\begin{array}{c}\text { Gdansk, Radom, Gliwice } \\
0.0499 \mathrm{~ms}\end{array}$ \\
\hline $\mathrm{C4}$ & Bologna & Cagliari, Milan & Rome, Milan, Cagliari \\
\hline C5 & $\begin{array}{l}0.0948 \mathrm{~ms} \\
\text { Evere } \\
0.0214 \mathrm{~ms}\end{array}$ & Evere, Louvain-la-Neuve & $\begin{array}{c}0.0433 \mathrm{~ms} \\
\text { Evere, Louvain-la-Neuve, Liege } \\
0.0140 \mathrm{~ms}\end{array}$ \\
\hline C6 & $\begin{array}{l}\text { Kirchberg } \\
0.0023 \mathrm{~ms}\end{array}$ & $\begin{array}{c}\text { Kirchberg, Ettelbruck } \\
0.0014 \mathrm{~ms}\end{array}$ & $\begin{array}{c}\text { Kirchberg, Esch-sur-Alzette, Diekirch } \\
0.0010 \mathrm{~ms}\end{array}$ \\
\hline $\mathrm{C} 7$ & $\begin{array}{l}\text { Vienna } \\
0.0901 \mathrm{~ms}\end{array}$ & $\begin{array}{l}\text { Vienna, Sofia } \\
0.0617 \mathrm{~ms}\end{array}$ & $\begin{array}{c}\text { Vienna, Sofia, Dornbirn } \\
0.0523 \mathrm{~ms}\end{array}$ \\
\hline $\mathrm{C8}$ & $\begin{array}{l}\text { Lausanne } \\
0.0351 \mathrm{~ms}\end{array}$ & $\begin{array}{c}\text { Lausanne , Zurich } \\
0.0251 \mathrm{~ms}\end{array}$ & $\begin{array}{l}\text { Lausanne, Zurich, Kreuzlingen } \\
0.0162 \mathrm{~ms}\end{array}$ \\
\hline C9 & $\begin{array}{l}\text { London } \\
0.0486 \mathrm{~ms}\end{array}$ & $\begin{array}{l}\text { London, Warrington } \\
0.0241 \mathrm{~ms}\end{array}$ & $\begin{array}{c}\text { London, Warrington, Glasgow } \\
0.0144 \mathrm{~ms}\end{array}$ \\
\hline C10 & $\begin{array}{l}\text { Madrid } \\
0.1100 \mathrm{~ms}\end{array}$ & $\begin{array}{l}\text { Madrid, Coimbra } \\
0.0588 \mathrm{~ms}\end{array}$ & $\begin{array}{l}\text { Madrid, Coimbra, Barcelona } \\
0.0452 \mathrm{~ms}\end{array}$ \\
\hline & & WORST- & CASE LATENCY \\
\hline Cluster & $\mathbf{K}=\mathbf{1}$ & $\mathbf{K}=\mathbf{2}$ & $\mathbf{K}=\mathbf{3}$ \\
\hline Co & $\begin{array}{c}\text { Copenhagen } \\
0.3744 \mathrm{~ms}\end{array}$ & $\begin{array}{l}\text { Frankfurt, Riga } \\
0.1865 \mathrm{~ms}\end{array}$ & $\begin{array}{c}\text { Copenhagen, Hannover, Riga } \\
0.1469 \mathrm{~ms}\end{array}$ \\
\hline C1 & $\begin{array}{c}\text { Amsterdam } \\
0.0574 \mathrm{~ms}\end{array}$ & $\begin{array}{l}\text { Zwolle, Eindhoven } \\
0.0417 \mathrm{~ms}\end{array}$ & $\begin{array}{c}\text { Nijmegen, Leeuwarden, Tilburg } \\
\text { 0.0347 ms }\end{array}$ \\
\hline C2 & $\begin{array}{l}\text { Limoges } \\
0.2522 \mathrm{~ms}\end{array}$ & $\begin{array}{l}\text { Rouen, Marseille } \\
0.1698 \mathrm{~ms}\end{array}$ & $\begin{array}{c}\text { Paris, Nantes, Cadarache } \\
0.1413 \mathrm{~ms}\end{array}$ \\
\hline C3 & $\begin{array}{l}\text { Lodz } \\
0.1582 \mathrm{~ms}\end{array}$ & $\begin{array}{c}\text { Gdansk, Gliwice } \\
0.1141 \mathrm{~ms}\end{array}$ & $\begin{array}{l}\text { Gdansk, Lodz, Bielsko-Biala } \\
0.0961 \mathrm{~ms}\end{array}$ \\
\hline $\mathrm{C} 4$ & $\begin{array}{l}\text { Rome } \\
0.1917 \mathrm{~ms}\end{array}$ & $\begin{array}{l}\text { Rome, Milan } \\
0.1490 \mathrm{~ms}\end{array}$ & $\begin{array}{c}\text { Cagliari, Rome, Milan } \\
0.1047 \mathrm{~ms}\end{array}$ \\
\hline C5 & $\begin{array}{l}\text { Vilvoorde } \\
0.0546 \mathrm{~ms}\end{array}$ & $\begin{array}{l}\text { Antwerpen, Namur } \\
0.0338 \mathrm{~ms}\end{array}$ & $\begin{array}{c}\text { Antwerpen, Namur, Evere } \\
0.0313 \mathrm{~ms}\end{array}$ \\
\hline C6 & $\begin{array}{l}\text { Kirchberg } \\
0.0075 \mathrm{~ms}\end{array}$ & $\begin{array}{l}\text { Hollerich, Diekirch } \\
0.0044 \mathrm{~ms}\end{array}$ & $\begin{array}{c}\text { Diekirch, Esch-sur-Alzette, Limpertsberg } \\
0.0026 \mathrm{~ms}\end{array}$ \\
\hline C7 & $\begin{array}{l}\text { Budapest } \\
0.3211 \mathrm{~ms}\end{array}$ & $\begin{array}{l}\text { Vienna, Sofia } \\
0.1458 \mathrm{~ms}\end{array}$ & $\begin{array}{l}\text { Vienna, București, Athina } \\
0.1429 \mathrm{~ms}\end{array}$ \\
\hline C8 & $\begin{array}{l}\text { Lausanne } \\
0.0642 \mathrm{~ms}\end{array}$ & $\begin{array}{l}\text { Bern, Kreuzlingen } \\
\quad 0.0422 \mathrm{~ms}\end{array}$ & $\begin{array}{c}\text { Bern, Kreuzlingen, Manno } \\
0.0403 \mathrm{~ms}\end{array}$ \\
\hline C9 & $\begin{array}{l}\text { Warrington } \\
0.0983 \mathrm{~ms}\end{array}$ & $\begin{array}{l}\text { Warrington, Telehouse } \\
0.0822 \mathrm{~ms}\end{array}$ & $\begin{array}{c}\text { Glasgow, Warrington, Reading } \\
0.0311 \mathrm{~ms}\end{array}$ \\
\hline C10 & $\begin{array}{l}\text { Madrid } \\
0.2183 \mathrm{~ms}\end{array}$ & $\begin{array}{l}\text { Madrid, Lisboa } \\
0.1404 \mathrm{~ms}\end{array}$ & $\begin{array}{c}\text { Lisboa, Sevilla , Valencia } \\
0.0842 \mathrm{~ms}\end{array}$ \\
\hline
\end{tabular}



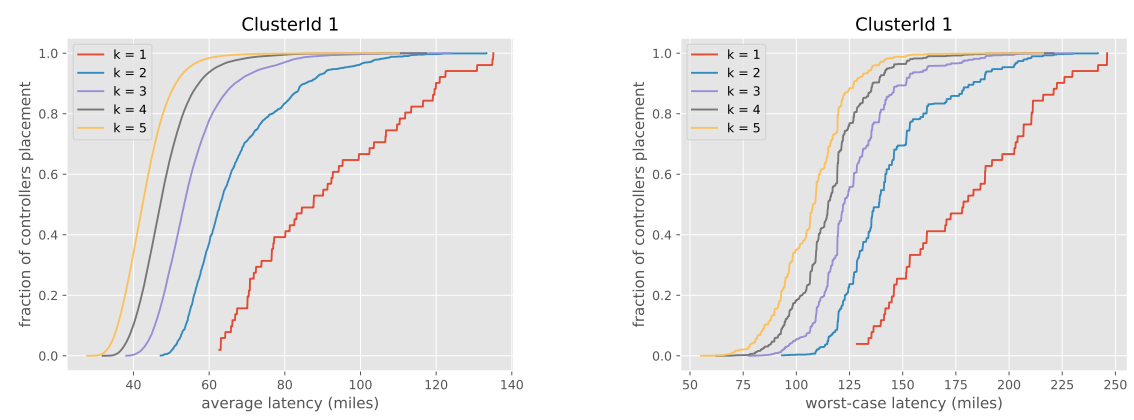

Fig. 3. Optimal latency CDFs for all possible controller combination placements for average-case latency (left) and worst-case latency (right)
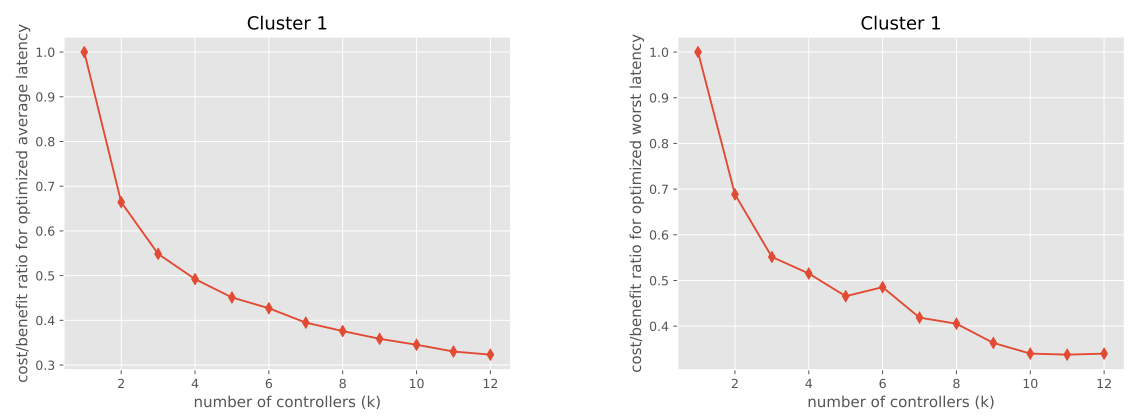

Fig. 4. Benefit to Cost Ratio for average-case latency (left) and worst-case latency (right)

\subsection{First Optimization: Clustering-based}

The latency between Domain Controller (DC) and Switches Fig.3 shows the Cumulative Distribution Function (CDF) for all possible combination placements of the controllers for cluster 1 by utilising a brute-force approach. The best placement corresponds to the lower latency for each value of $k$. We use cluster 1 (Netherlands) as an example. The optimum solution when the number of Domain Controllers (DCs) is one $(\mathrm{k}=1)$ given an average latency of $\left(L_{c s-a v g}=\right.$ $0.0280 \mathrm{~ms}$ ) and the optimal controller location is in Utrecht. For the worst-case latency is $\left(L_{c s-\text { worst }}=0.0574 \mathrm{~ms}\right)$ and the placement is in Amsterdam.

The latency between Domain Controller (DC) and their associated switches decreases gradually as more controllers added in the network.

Table 2 summarize the optimum latency and the locations of controllers outcomes of the optimization model for each cluster when the number of Domain Controllers (DCs) is varied from one to three. Running the optimization model is defined in Section2. 
Benefit to Cost Ratio The goal of this metric is to decide if more controllers should be deployed. Adding a new controller reduces latency but adds cost. The Benefit to Cost Ratio is defined as:

$$
\left(l_{1} t_{1} / l a t_{k}\right) / k
$$

Where $l a t_{1}$ represents the optimal latency with a single controller, $l_{a t} t_{k}$ represents the latency with $k$ controllers. The reason to divide by $k$ to take into account in the cost of adding controllers [6].

Fig.4 shows the Benefit to Cost Ratio of deploying Domain Controllers (DCs) when the number of controllers is increasing from 1 to 12 .

In this paper, we define a rule to make an easier way to determine how many controllers we need. The minimum number of controllers required corresponds to the latency $($ lat 1$) / 2$. Looking for a reduction of the latency when $\mathrm{k}=1$ to a half, we conclude that three to four controllers are suitable to optimize the average latency and four to five controllers for worst-case latency. We found out that the number of controllers is similar for the other clusters.

\subsection{Second Optimization: Master Controllers (MCs) Placement}

In the second stage of optimization, we generate the topology of a Virtual Network (VN) by using the physical underlying real topology interconnecting the Domain Controllers (DC) and switches. Dijkstra Algorithm is applied to compute distances and shortest paths between all pairs of Domain Controllers (DCs).

Fig.5 presents the latency between the Master Controller (MC) and Domain Controllers (DC). The optimal average latency is gradually decreasing by increasing the number of Domain Controllers (DCs) Fig.5(left).

On the other hand, results in the worst scenario indicates the instability in some optimal solutions due to the limitation of the data links between two points in the real world topology despite the fact others are relatively similar to the average scenario Fig.5(Right).

The results of the second stage of optimization show that a single Master Controller (MC) provides the best performance on the Western European NRENs topology.

The solution of the second optimization gives us a single master controller and it is the optimal solution for the control plane. This means that we do not need the third optimization to apply for this particular topology.

The Master Controller (MC) is the main controller that manages Domain Controllers (DCs) in different countries, while the Domain Controller (DC) manages switches in cities in the same country or neighbourhood countries.

Fig.6 shows the particular case where four Domain Controllers (DCs) per cluster are deployed. This means that the total number of Domain Controllers (DCs) is 44 for the second optimization. Then, we build a virtual network topology (VN) for the 44 Domain Controllers (DCs). Dijkstra algorithm is implemented on the top of the physical underlying topology to determine the shortest 

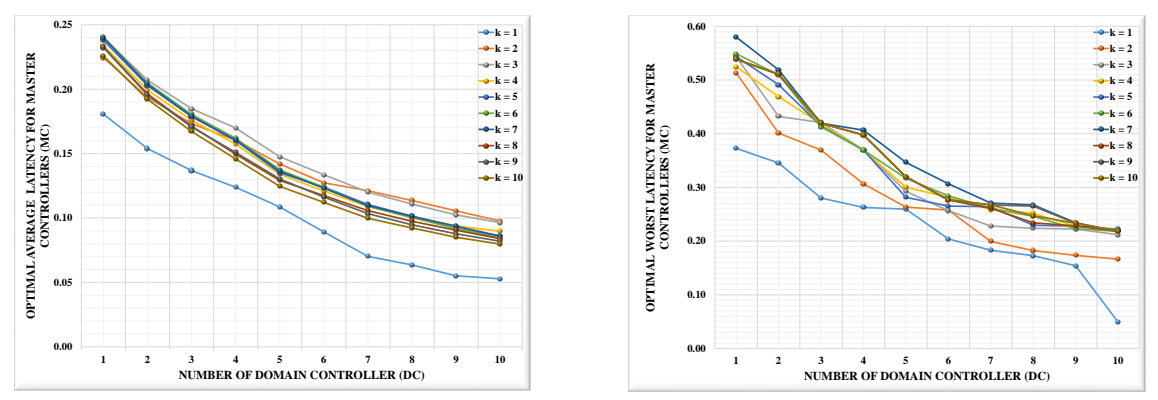

Fig. 5. The optimal solution for Second Optimization for average-case latency (ms) (left) and worst-case latency (ms) (right)
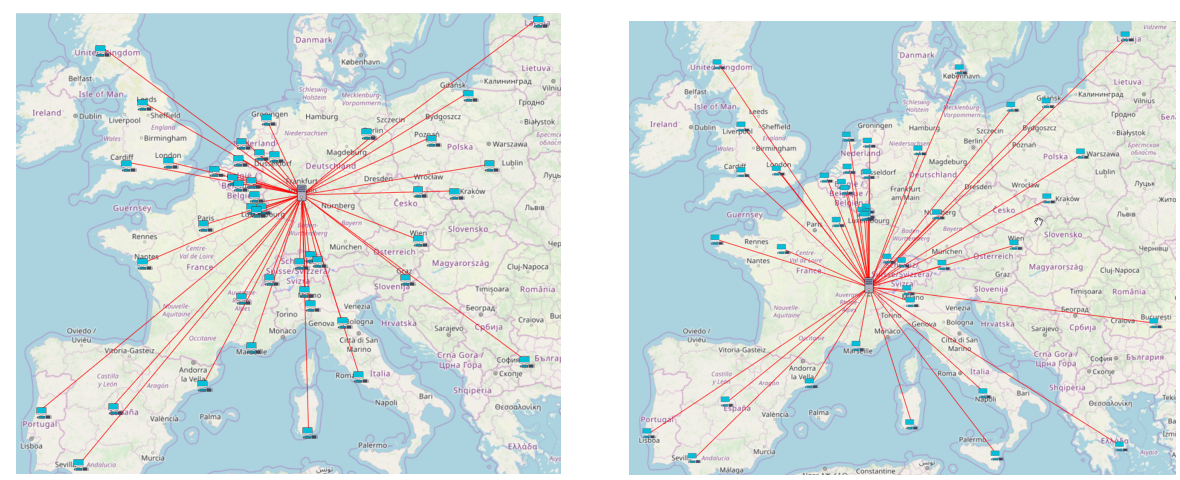

Fig. 6. The particular case for Master controller location for average-case latency (left) and worst-case latency (right)

path between each pair of Domain controller (DC). Then, we run the optimization to find the best placement of the Master Controller (MC).

For the average-case latency, Fig.6(left) shows the Master Controller (MC) is located in Frankfurt city (Germany) and its central geographical location. In addition, another significant factor is the massive connectivity between Frankfurt city and other cities in the network. Fig.6(right) illustrates the results of the second optimization for the worst-case scenario. The Master Controller (MC) placement is CERN (Switzerland). CERN is located at Geneva.

\subsection{Applicability case study}

In this section, we compare the results obtained when applying the hierarchical controller placement against the case of a single level controller placement.

The main point is to demonstrate the feasibility of deploying multiple levels of controllers in very large networks composed of autonomous networks, as the case 

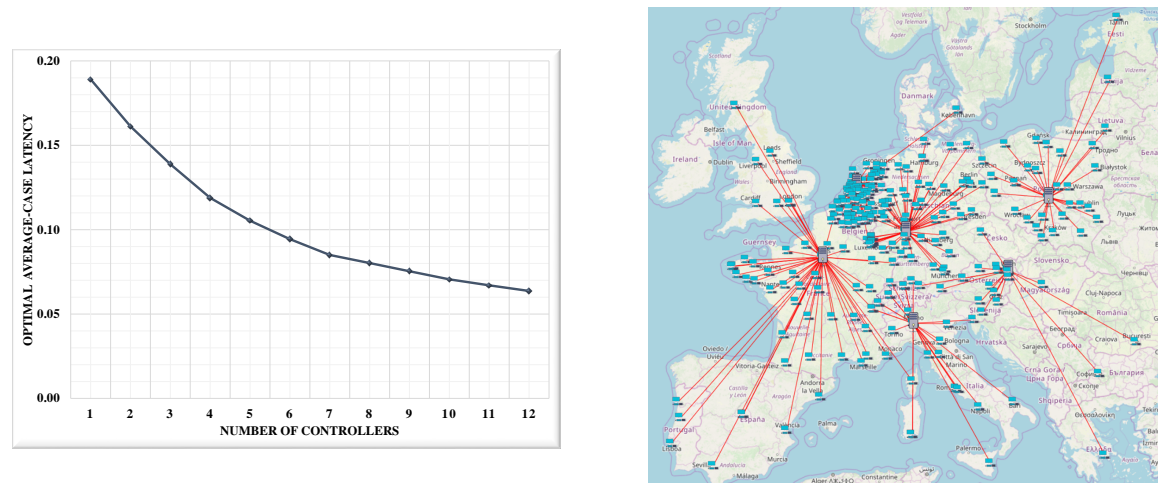

Fig. 7. The Optimal Average Latency of the Whole Western European NRENs for Plain Control Case (ms) (left) and The Whole Western European NRENs with Optimal Average Location when the number of controller is six $(k=6)$ for Plain Control Case (right)

of the European NRENs. Each autonomous network has its own management policy and coordinates with its neighbours. Let's consider the case of interdomain routing policies or end-to-end traffic engineering policies. Each network manager wants to have a close control on how the policies are applied within its network and, at the same time, wishes to disclose a limited amount of information and details to its neighbours. Domain Controllers are the appropriate approach for the application of intra-domain rules while Master Controllers may deal with the inter-domain rules.

For this comparison, we consider the average latency case. The whole set of NRENs is considered as a unique network and a single optimization step results in the optimal location of the controllers. Following the same optimization function and approach presented in Section II the average latency of one single controller is $0.1807 \mathrm{~ms}$ (this is lat1) and using the same rule to determine the best number of controllers needed (looking for lat1/2) results in six controllers (with an average latency of $0.0894 \mathrm{~ms}$ ). Fig.7(left) shows all results for optimal average latency of the Whole Western European NRENs without applying the Multi-level Hierarchical Control Plane methodology when the number of controllers is varied from 1 to 12. Fig.7(right) presents the map with the location of the six controllers [(Vienna, Austria), (Amsterdam, Netherlands), (Frankfurt, Germany), (Milan, Italy), (Paris, France) and (Lodz, Poland)]. This result is referred as plain control plane.

The results obtained applying the hierarchical method gives that three Domain Controllers per cluster is the best option using the decision rule presented. Then, the second optimization results in a single Master Controller, which is placed in Frankfurt for the average latency case. This case is referred as Hierarchical Control Plane. 
Comparing the results, for the plain control the average latency is 0.0894 $\mathrm{ms}$. For the hierarchical plane, there is an average latency between the Domain Controller and Switch is about $0.03576 \mathrm{~ms}$ and the average latency between Master Controller and Domain Controller of $0.0894 \mathrm{~ms}$. As most of the decisions may be made by the Domain Controllers the average latency is better with the hierarchical control but for some higher-level decisions, the average latency will be about the same than in the plain control approach. This is true only if all information from all the autonomous networks is shared and sent to the controllers in the plain control case.

From a practical point of view and real feasibility, we claim that the hierarchical control case allows detailed control within each domain and that the extra latency that may occur for some decisions made at the Master Controller will be compensated by the gain in management flexibility and autonomy.

\section{Conclusion}

Placement of controllers has received significant attention in recent years in the large scale networks. In this study, we minimize the latency between controllers and their associated switches.

This work presents a Multilevel Hierarchical Control Plane Architecture in SDN. The performance of this technique is evaluated with the real-world networks of the Western European NRENs). The topologies are taken from the Internet Topology Zoo collection. This is the first kind of recent CPP study is applied to the National Research and Education Network (NREN) in Europe.

The design methodology is performed from the bottom to the top because we consider the administrative boundaries; each NREN has its own management policy and this is the main constraints. For this reason, the real network topology (Western European NRENs) is divided into clusters by using a countries-based approach. The main contributions are the following.

As a result of the first stage optimization, we provide a rule to make an easier way to determine how many controllers we need. As a result, we found out that the number of Domain Controllers (DCs) is similar for each cluster based on the metrics; K-means and K-centre, respectively. Generally, three to four Domain Controllers (DCs) are required in case of average latency; or four to five Domain Controllers (DCs) in the worst-case latency scenario.

Second, the optimal placement of master controllers (MC) is proposed during the second phase of optimization. Results show that a single Master Controller (MC) is sufficient to manage the entire network to achieve the best performance in respect of this particular case study.

The overall contribution is the methodology presented to define a multi-level control plane as an iterative optimization problem. For this particular topology the results show that a third level is not needed. Other large topologies with different constraints may need a third level in the control plane. 
All datasets and results will be made available for research purposes. Further research is progress to consider the reliability and capacity constraints of the Hierarchical Controller Placement Problem (HCPP).

\section{Acknowledgements}

This work was funded by the Spanish Ministry of Economy and Competitiveness under contract TEC2017-90034-C2-1-R (ALLIANCE).

\section{References}

1. Nunes, B. A. A., Mendonca, M., Nguyen, X. N., Obraczka, K., \& Turletti. A survey of software-defined networking: Past, present, and future of programmable networks. IEEE Communications Surveys and Tutorials, Volume: 16, Issue: 3, Page(s): 1617 - 1634, Third Quarter 2014.

2. F. Bannour, S. Souihi, and A. Mellouk, "Distributed SDN Control: Survey, Taxonomy, and Challenges," IEEE Communications Surveys Tutorials, vol. 20, no. 1, pp. 333-354, Firstquarter 2018.

3. J. F. Kurose, Computer Networking: A Top-Down Approach Featuring the Internet. London, U.K.: Pearson, 2005.

4. G. Wang, Y. Zhao, J. Huang, and Y. Wu, "An Effective Approach to Controller Placement in Software Defined Wide Area Networks," IEEE Trans. Netw. Serv. Manag., vol. 15, no. 1, pp. 344-355, 2018.

5. V. Arya, N. Garg, R. Khandekar, A. Meyerson, K. Munagala, and V. Pandit, "Local search heuristics for k-median and facility location problems," Conf. Proc. Annu. ACM Symp. Theory Comput., vol. 33, no. 3, pp. 21-29, 2001.

6. Heller, B., Sherwood, R., McKeown, N.: The controller placement problem. In:ACM SIGCOMM Workshop on Hot Topics in Software Defined Networking, pp. 7-12 (2012).

7. F. Yeung, "Internet 2: scaling up the backbone for R\&D," IEEE Internet Computing, 1997, vol. 1, no. 2, pp. 36-37.

8. G. Wang, Y. Zhao, J. Huang, Q. Duan and J. Li, "A K-means-based network partition algorithm for controller placement in software defined network," 2016 IEEE International Conference on Communications (ICC), Kuala Lumpur, 2016, pp. 1-6, doi: 10.1109/ICC.2016.7511441.

9. K. S. Sahoo, B. Sahoo, R. Dash and M. Tiwary, "Solving Multi-controller Placement Problem in Software Defined Network," 2016 International Conference on Information Technology (ICIT), Bhubaneswar, 2016, pp. 188-192, doi: 10.1109/ICIT.2016.047.

10. A. K. Singh and S. Srivastava, "A survey and classification of controller placement problem in SDN," Int. J. Netw. Manag., vol. 28, no. 3, 2018.

11. Hock, D., Hartmann, M., Gebert, S., Jarschel, M., Zinner, T., Tran-Gia, P.: Paretooptimal resilient controller placement in SDN-based core networks. In: International Teletraffic Congress, pp. 1-9 (2013)

12. T. Hu, Z. Guo, P. Yi, T. Baker and J. Lan, "Multi-controller Based SoftwareDefined Networking: A Survey," in IEEE Access, vol. 6, pp. 15980-15996, 2018, doi: 10.1109/ACCESS.2018.2814738. 
13. H. Kuang, Y. Qiu, R. Li, and X. Liu, "A Hierarchical K-means Algorithm for Controller Placement in SDN-based WAN Architecture," in 10th International Conference on Measuring Technology and Mechatronics Automation, 2018.

14. M. Karakus and A. Durresi, "A survey: Control plane scalability issues and approaches in software-defined networking (SDN)," Comput. Netw., vol. 112, pp. 279-293, Jan. 2017

15. S. Knight, H. X. Nguyen, N. Falkner, R. Bowden, and M. Roughan, "The internet topology zoo," IEEE J. Sel. Areas Commun., vol. 29, no. 9, pp. 1765-1775, 2011.

16. IBM ILOG. Cplex optimizer. http://www 01.ibm.com/software/commerce/optimization/ cplex-optimizer; 2012. 